\title{
A COMBINED APPROACH FOR TREATING MULTI-OBJECTIVE PROGRAMMING PROBLEM
}

\author{
Ahmed A. Elsawey, Taghreed A. Hassan \\ Basic Engineering Science Department, Faculty of Engineering, \\ Minoufiya University, Shebin El-Kom, Egypt
}

\begin{abstract}
The main purpose of this paper is to introduce a comprehensive study of vector optimization problems (VOP). A new combined approach is developed for characterizing the efficient solutions of (VOP). This approach combines the characteristics of both Tchebycheff norm approach and $\mathrm{K}_{\text {_th }}$ objective Lagrangian approach to form an improved scalarizing method for treating nonlinear multiobjective optimization problems. The Combined procedure is sensible because the Tchebycheff norm approach possesses complementary distinguishing characteristics for $\mathrm{K}$-th objective Lagrangian approach. The theorems which characterize the efficient solutions of (VOP) in terms of this new combined approach are introduced. Also, some of the basic notions in parametric convex programming are redefined and analyzed for the new combined approach.

الغرض من هذا البحث هو دراسة هنهجية النمج بين خصائص الطرق المستخدمة لعلاج مشاكل الأمثلية الاتجاهية.

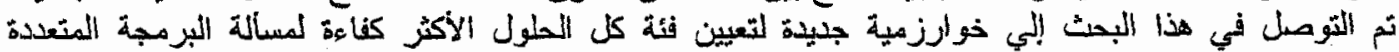

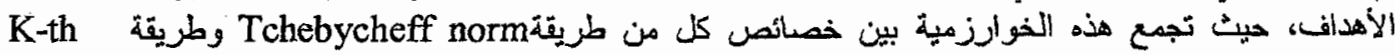
objective Lagrangian

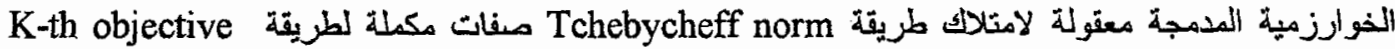

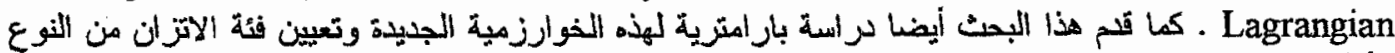
الأول.
\end{abstract}

Keywords Vector optimization, combined approaches, Tchebycheff approach, Lagrangian approach, Convex programming.

\section{INTRODUCTION}

A variety of scalarization methods for finding efficient solutions of multiple objective programs (MOPs) have been developed over the last two decades. Some of the methods were designed specifically for linear problems and others work well only on problems with concave objective functions and a convex feasible region [1].

Scalarization meaning that the multiobjective problem is converted into a single or a family of single objective optimization problems [2].

Three requirements are set to any scalarizing function:

1. it can cover any Pareto optimal solution,

2. every solution is Pareto optimal,

3. its solution is satisfactory if the aspiration levels used are feasible (if the scalarization function is based on aspiration levels).

Unfortunately, no scalarizing function can satisfy all three requirements [2].
One of a few methods that can generate efficient solutions of general MOPs is the Tchebycheff scalarization that selects an efficient solution based on the minimization of the Tchebycheff distance of the objective functions from an ideal point [3]. Among the first who proposed to apply the Tchebycheff norm to MOPs in the early seventies were YU, Zeleny, and Bowman [4]. In the eighties, that direction of research was explored by Wierzbicki, Ecker and Shoemaker, Choo and Atkins [5]; and many others. In parallel, the theorey of generalized Lagrangian duality in single objective mathematical programming has been developed as a means for resolving a duality gap that may exist for nonconcave problems. Specific generalized Lagrangian functions were introduced by Roode. [Gould] proposed a multiplier function and Nakayama et al. contiued in that direction by adding to the reqirements of the multiplier function [1]. As the generalized Lagrangian duality theory plays a major role in the analysis and solution of general 
constrained single objective nonlinear programs, it also turned out to be helpful in generating efficient solutions of nonconcave multiobjective problem. TenHuisen and Wiecek proposed a framework for developing generalized Lagrangian-type scalarizing functions for nonconcave multiobjective problem [1].

Although numerous interactive procedures have been suggested, none has emerged as a clearly preferred approach, given that managers have decidedly different decision making styles and these styles result in different approaches to problem solving. Recently, researchers introduced the concept of the unified algorithm which links various approaches in a way that makes use of the advantages of the linked approaches and avoided their disadvantages [6]. Wendell [7] combined the weighting problem and the constraint problem to introduce another form of scalar optimization methods, which is called the hybrid approach. Ragab [8] used both of the weighting problem and constraint problem. Hegazy [3] combined the characteristics of both the generalized Tchebycheff norm method and the constraint method lo introduce a modified hybrid approach. Hussein [9] combined the weighted norm problem and the constraint problem. Hamada [10] combined the characteristics of both the Lagrangian problem and the constraint problem. Awad [11] combined the generalized Tchebycheff norm and the proper equality constraint. Steuer, and Silverman, and Whisman. [12] introduced a unified approach called a combined Tchebcheff / aspiration critetion vector interactive multiohjective programming procedure. Kassem [13] proposed two unified approaches. The first approach is a general unified parametric approach. The second approach is a general unified nonlinear goal programming approach. Gardiner and Steuer [14] suggested unified procedures linking various approaches and allowing users to switch between methodologies. Tarrad [15] developed an approach that solves generalized goal programming problems using visual interactive method. Kanaya [16] proposed a unified interactive approach for treating nonlinear multiobjective optimization problems in fuzzy environment. Abu elnaga [17], developed an interactive combined approach between the I-SHOT and NIMBUS techniques. Khdour [6], developed a unified approach (ARPRDA) between the Attainable Reference Point method and the Reference Direction method

With respect to the multiple objective program:

(VOP):

$$
\begin{gathered}
\operatorname{Min} F(x)=\left\{f_{1}(x) \ldots f_{1}(x) \ldots f_{k}(x)\right\} \\
\text { s.t. } \quad x \in S
\end{gathered}
$$

We will combine the characteristics of both Tchebycheff norm approach and K-th objective Lagrangian approach to form an improved scalarizing approach for treating nonlinear multiobjective optimization problems, and introduce a parametric study.

\section{COMPONENT APPROACHES}

\subsection{Different metrics [2]}

The ideal objective vector is used as a reference point and $L_{P}$-metrics are used for measuring the distances. In this case the $L_{P}$-problem to be solved is:

$$
\begin{array}{ll}
\text { minimize } & \sum_{i=1}^{k}\left(\left|f_{i}(x)-z_{i}^{*}\right|^{p}\right)^{1 / p} \\
\text { subject to } & x \in S
\end{array}
$$

If $P=\infty$, the metric is $L \infty$ or Tchebycheff and the $L \infty$ or Tchebycheff problem is solved:

$$
\begin{aligned}
& \text { minimize } \max _{i=1, \ldots, k}\left(\left|f_{i}(x)-z_{i}^{*}\right|\right) \\
& \text { subject to } x \in s
\end{aligned}
$$

The above problem is nondifferentiable. Instead, we have the problem of $\alpha \in R$ and $x \in R^{n}$ variables:

minimize $\alpha$

$$
\begin{aligned}
& \text { subject to } \quad \alpha \geq f_{i}(x)-z_{i}^{*}, \\
& \text { for all } i=1, \ldots, k \\
& \qquad x \in s
\end{aligned}
$$

2.2 Theoretical results [2].

Some theoretical results concerning this method can be summarized as:

Theorem 2-1: The solution of $L p$-problem is Pareto optimal.

Theorem 2-2: The solution of Tchebycheff problem is weakly Pareto optimal.

Theorem 2-3: Tchebycheff problem has at least one Pareto solution.

Hegazy [3] Showed that the generalized Tchebycheff norm problem takes this form:

$$
\min _{x \in S} \max _{j} \beta_{j}\left|f_{j}(x)-u_{j}^{*}\right|
$$

where $\beta \in R_{+}^{m}, R_{+}^{m}$ is the positive orthant of $\mathrm{R}^{\mathrm{m}}, \mathrm{U}^{*}$ is an ideal target,

$$
\begin{aligned}
& u_{j}^{*}=\tilde{f}_{j}-\delta, j=1,2, \ldots, m, \\
& \text { where } \tilde{f}_{j}=\min _{x \in S}(x)
\end{aligned}
$$

and $\delta$ is a small positive number

and $\beta$ is normalized by the condition $\beta_{1}=1$, then the problem has the following forms:

$$
\left(\mathrm{P}^{0}(\beta)\right) \min _{\mathrm{x} \in \mathrm{s}} \max _{\mathrm{j}} \beta_{\mathrm{j}}\left[\mathrm{f}_{\mathrm{j}}(\mathrm{x})+\delta-\overline{\mathrm{f}}_{\mathrm{j}}\right]
$$

or equivalent 
$(\mathrm{P}(\beta)) \quad \min \mathrm{Z}$

subject to:

$$
\begin{aligned}
& -\mathrm{z}+\beta_{\mathrm{j}}\left|\mathrm{f}_{\mathrm{j}}(\mathrm{x})+\delta-\overline{\mathrm{f}}_{\mathrm{j}}\right| \leq 0, j=1,2, . ., m, \\
& g_{k}(x) \leq 0, k=1,2, . ., r .
\end{aligned}
$$

2.3 Characterizing efficient solutions for MOP in terms of the optimal solutions of $P(\beta)$ [3].

Theorem: $x^{*}$ is an efficient solution of MOP if it is optimal solution to $P(\beta)$ for some $\beta=\beta^{*}$.

Definition: the efficient set is uniformaly dominant if for every nonefficient point $\bar{x}$, there exists an efficient one $x^{*}$ such that $f_{i}(\bar{x}) \prec f_{i}\left(x^{*}\right)$ for alli.

Theorem: If the efficient set is uniformaly dominant, then all solutions to $P(\beta)$ are efficient solutions of MOP.

2.4 The Kth-objective Lagrangian Method Hegazy [3] has characterized the efficient solutions of MOP in terms of the optimal solutions of the following form:

$$
\begin{aligned}
& \left(\mathrm{P}_{\mathrm{k}}(u)\right) \min _{x \in S}\left[f_{k}(x)+\sum_{j \neq k} u_{j} f_{j}(x)\right] \\
& \text { where; } u \in U \equiv\left\{\begin{array}{l}
\left(u_{1}, u_{2}, \ldots, u_{k-1}, u_{k+1}, \ldots, u_{m}\right) \\
l u_{j} \geq 0 \text { for each } j \neq k
\end{array}\right\}
\end{aligned}
$$

is the set of Lagrangianmultiplier

2.5 Characterizing efficient solutions for MOP in terms of the optimal solutions of $\mathbf{P}_{\mathbf{k}}(\mathbf{u})$ [3].

Definition:

Let $\psi_{\mathrm{i}}(\gamma)=\inf \left\{f_{i}(x) / G(x) \leq \gamma\right\}$

$, i=1,2, . ., m, \gamma \in \stackrel{m}{R}$,

then problem MOP is said to be

stable if $\psi_{\mathbf{i}}(0)$ are finite,

and there exist scalars $L_{i}$ such that

$\frac{\psi_{\mathrm{i}}(0)-\psi_{\mathrm{i}}(\gamma)}{\|\gamma\|} \leq L_{i}$, for all $\gamma \neq 0, i=1,2, \ldots, m$

Haimes [18] discusses the necessary conditions for efficiency which are expressed in terms of solutions of $\mathrm{P}_{\mathrm{k}}(\mathrm{u})$ in the following theorems:

Theorem [18] :

Assuming convexity assumption, if $\mathrm{x}^{*} \in X^{*}$

and for any given $K$,

1) $P_{k}(\varepsilon)$ is stable.

2) all $f_{j}, j=1,2, \ldots, m$ and $g_{r}, r=1,2, \ldots, k$

are faithfully convex with $S$.

3) the constraint $s$ of $P_{k}\left(\varepsilon^{*}\right)$ satisfy karlin's constraint qualificafon; or

4) the constraints of $P_{k}\left(\varepsilon^{*}\right)$ satisfy

regularity assumptions of $\mathrm{x}^{*}$.

Then there exist $u \in U_{k}$ such that $\mathrm{x}^{*}$ solves $P_{k}(u)$ Theorem [3]:

$\mathrm{X}^{*}$ is an efficient solution of MOP if for some $\mathrm{k}$ there exists $u \in U_{k}$ such that $x^{*}$ solves $P_{k}(u)$ and if either; 1. $u_{j}>0$ for all $j \neq k$, or

2. $x^{*}$ is the unique minimizer of $P_{k}(u)$.

The limitation on $P_{k}(u)$ approach is that not all efficient solutions of MOP are optimal solutions of $P_{k}(u)$ for some $u \geq 0$, and vice versa. Moreover, imposed conditions that allow this to be true are somewhat complicated [3].

\section{COMBINED APPROACH}

In this section the formulation of a new combined approach will be discussed with a parametric study. This approach is deduced by combining the generalized Tchebycheff norm approach together with the k-th objective Lagrangian approach.

Problem formulation

$(p(\beta, U)): \operatorname{Min}\left[Z+f_{k}(x)+\sum_{j \neq k} u_{j} f_{j}(x)\right]$

subject to

$-z+\beta_{i}\left[f_{i}(x)+\delta-\bar{f}_{i}\right] \leq 0, \quad i=1,2, . ., m$

, $(\mathrm{i}=\mathrm{j}+\mathbf{k}$ all objective functions)

$\mathrm{g}_{\mathrm{S}}(\mathrm{x}) \leq 0, \mathrm{~s}=1,2, \ldots, \mathrm{r}$

where; $u \in U \equiv\left\{\begin{array}{l}\left(u_{1}, u_{2}, \ldots ., u_{k-1}, u_{k+1}, \ldots ., u_{m}\right) \\ / u_{j} \geq 0 \text { for each } j \neq k\end{array}\right\}$

is the set of Lagrangianmultiplier

note that there exists a parameter in the objective function and an other in the constraints.

Definition: The problem $P(\beta, U)$ is stable.

Let $\psi_{i}(\gamma)=\inf \left\{\left(f_{i}(x)+Z\right) / G(x) \leq \gamma\right\}, i=1,2, \ldots, m, \underset{R}{m}$, then problemMOP is said to be stable

if $\Psi_{\mathrm{i}}(0)$ are finite,

and thereexist scalarsi $i$ such that

$\frac{\psi_{i}(0)-\psi_{i}(\gamma)}{\|\gamma\|} \leq L_{i}$, for all $\gamma \neq 0, i=1,2, \ldots, m$

3.1 Characterizing efficient solutions for VOP in terms of the optimal solutions of $P(\beta, U)$

The following Theorems establish the relation between the efficient solutions of MOP and the optimal solution of $P(\beta, U)$ problem. 
Theorem [3]: $x^{*}$ is an efficient solution of MOP iff $x^{*}$ is an optimal solution of $P(\beta, U)$ for any given $\beta>$ 0 and for some $u \in R^{(m-1)}$ for which $P(\beta, U)$ is feasible.

Proof: See Hegazy [3].

Theorem [3]: Assume that one of the following holds:

I) $P(\beta, u)$ is stable, the set of the constrains is convex, and $f_{i}(x), i=1,2, \ldots \ldots \ldots . . m$ are convex $\mathrm{m}$

functions on $\mathrm{R}$ or

II). All $\mathrm{f}_{\mathrm{i}}(\mathrm{x}), \mathrm{i}=1,2, \ldots \ldots \ldots \ldots \mathrm{m}$ and $\mathrm{g}_{\mathrm{k}}(\mathrm{x})$,

$k=1,2, \ldots r$ are faithfully convex on $\mathrm{m}_{\mathrm{m}}^{\mathrm{m}}$, then $\mathrm{x}^{*}$ of VOP $=\phi$ if $\phi\left(u^{*}\right)=-\infty$. Where $x^{*}$ is the set of all efficient solution of VOP.

Proof: See Hegazy [3].

3.2 Characterization of the set of feasible parameters

Definition [3]: The set of feasible parameters for problem $P(\beta, u)$ denoted by $Q$, is defined by: $Q=\left\{(\beta) \in R^{m} / N(\beta) \neq \phi\right\}$, the set $Q$ is nonempty.

\section{LEMMA:}

If $N\left(\beta^{1}\right) \cap N\left(\beta^{j}\right) \neq \phi$ for all $L, J$ and $\beta_{i} \succ 0$,

$$
\mathrm{i}=1,2, . ., \mathrm{m}
$$

Then the set $Q$ is convex.

Proof: See Hegazy [3].

Definition [19]: The solvability set of problem (VOP), which is denoted by $(G)$, is defined by:

$G=\left\{\begin{array}{l}(\beta) \in R^{m},(u) \in R^{m-1} \\ / \text { problem (VOP) has efficient solutions }\end{array}\right\}$

3.3 Characterization of the stability set of the first kind.

Definition [19]: Suppose that the problem $P(\beta, u)$ is solvable for $(\bar{\beta}, \overline{\mathrm{u}})$ with a corresponding optimal point $(\bar{z}, \bar{x})$, then the stability set of the first kind of $P(\beta, u)$ corresponding to $(\bar{z}, \bar{x})$, which is denoted by

$\mathrm{S}(\overline{\mathrm{x}})$, is defined by

$\mathrm{S}(\overline{\mathrm{x}})=\left\{\begin{array}{l}(\beta) \in \mathrm{R}^{\mathrm{m}},(u) \in R^{m-1} \\ /(\bar{x}) \text { is an efficient solutions of }(V O P), \\ \beta \succ 0, u \succ 0\end{array}\right\}$

From the assumption that problem $P(\beta, u)$ is stable, then there exist

$\bar{\lambda} \in \mathrm{R}^{\mathrm{m}}$ and $\bar{v} \in R^{r} \quad$ such that $(\bar{z}, \overline{\mathrm{x}})$

solves the following kuhn - Tucker conditions: $\sum_{i=1}^{m} \lambda_{i}=1$

$\frac{\partial Z}{\partial X_{\alpha}}+\frac{\partial f_{k}(\bar{x})}{\partial X_{\alpha}}+\sum_{j \neq k} \bar{u}_{j} \frac{\partial f_{j}(\bar{x})}{\partial X_{\alpha}}+$

$\sum_{i=1}^{m} \bar{\lambda}_{i}\left(\bar{\beta}_{i} \frac{\partial f_{i}(\bar{x})}{\partial X_{\alpha}}-\frac{\partial Z}{\partial X_{\alpha}}\right)+\sum_{s \notin l} \bar{v}_{s} \frac{\partial g_{s}(\bar{x})}{\partial X_{\alpha}}=0$

, $\alpha=1,2, \ldots, n+1$

where: $n$ (number of decision variables)

$$
\begin{aligned}
& -\mathrm{z}+\bar{\beta}_{\mathrm{i}}\left[\mathrm{f}_{\mathrm{i}}(\overline{\mathrm{x}})+\delta-\overline{\mathrm{f}}_{\mathrm{i}}\right] \leq 0, \quad i=1,2, . ., m \\
& ,(i=j+k \text { all abjective functions }) \\
& \quad g_{s}(\bar{x}) \prec 0, \quad s \in L \subset\{1,2, . ., r\} \\
& g_{s}(\bar{x})=0, \quad s \notin L \\
& \quad \lambda_{i}\left[\bar{\beta}_{\mathrm{i}}\left[\left[_{\mathrm{i}}(\bar{x})+\delta-\overline{\mathrm{f}}_{\mathrm{i}}\right]-\bar{z}\right]=0, i=1,2, . ., m\right. \\
& \bar{v}_{k} \geq 0, \quad \bar{\lambda}_{i} \geq 0,, \bar{\beta}_{\mathrm{i}} \succ 0 \\
& \text { Differentiate w.r.to } \mathrm{z}: \\
& 1-\lambda_{i}=0 \quad, i=1,2, \ldots, m
\end{aligned}
$$

Differentiate w.r.to $\mathrm{x}_{\mathbf{1}}$ :

$$
\begin{aligned}
& \frac{\partial f_{k}(\bar{x})}{\partial X_{1}}+\sum_{j \neq k} \bar{u}_{j} \frac{\partial f_{j}(\bar{x})}{\partial X_{1}}+\lambda_{i} \beta_{i} \sum_{i=1}^{m} \frac{\partial f_{i}(\bar{x})}{\partial X_{1}}+ \\
& \sum_{s=1}^{r} \bar{v}_{s} \frac{\partial g_{s}(\bar{x})}{\partial X_{1}}=0
\end{aligned}
$$

Differentiate w.r.to $x_{2}$ :

$$
\begin{aligned}
& \frac{\partial f_{k}(\bar{x})}{\partial X_{2}}+\sum_{j \neq k} \bar{u}_{j} \frac{\partial f_{j}(\bar{x})}{\partial X_{2}}+\lambda_{i} \beta_{i} \sum_{i=1}^{m} \frac{\partial f_{i}(\bar{x})}{\partial X_{2}}+ \\
& \sum_{s=1}^{r} \bar{\nu}_{s} \frac{\partial g_{s}(\bar{x})}{\partial X_{2}}=0
\end{aligned}
$$

Differentiate w.r.to $x_{n}$

$$
\begin{aligned}
& \frac{\partial f_{k}(\bar{x})}{\partial X_{n}}+\sum_{j \neq k} \bar{u}_{j} \frac{\partial f_{j}(\bar{x})}{\partial X_{n}}+\bar{\lambda}_{i} \bar{\beta}_{i} \sum_{i=1}^{m} \frac{\partial f_{i}(\bar{x})}{\partial X_{n}}+ \\
& \sum_{s=1}^{r} \bar{\nu}_{s} \frac{\partial g_{s}(\bar{x})}{\partial X_{n}}=0
\end{aligned}
$$

Let the above linear independent system of equations (14-23) be written in the following matrix form:

$\left[\begin{array}{llll}A & B & C & D\end{array}\right]\left[\begin{array}{c}1 \\ \bar{u}_{j} \\ \bar{\gamma}_{i} \\ \bar{v}_{S}\end{array}\right]=0$

where $\bar{\gamma}_{i}=\bar{\lambda}_{i} \bar{\beta}$

where $A$ is (s $\times 1)$ matrix, $B$ is (s $x(m-1))$ matrix, $C$ is $(\mathrm{s} \times \mathrm{m})$ matrix, $\mathrm{D}$ is (s $\mathrm{x} r)$ matrix, $\bar{u}_{j} \in R^{m-1}, \bar{u}_{j} \geq 0, \bar{u}_{j} \neq 0, \beta \in R^{m}, \beta \neq 0$,

$v \in R^{r}, v \geq 0, s \leq n$,

and $r$ is the cardinality of the set of active constraints. There are more than one linear program so that an equivalence can be found between the set of 
parameters corresponding to a certain optimal solution of any of them and the set $\mathrm{S}(\overline{\mathrm{X}})$

At $\bar{v}_{S}=0$

$\frac{\partial f_{k}(\bar{x})}{\partial X_{n+1}}+\sum_{j \neq k} \bar{u}_{j} \frac{\partial f_{j}(\bar{x})}{\partial X_{n+1}}+\bar{\lambda}_{i} \bar{\beta}_{i} \sum_{i=1}^{m} \frac{\partial f_{i}(\bar{x})}{\partial X_{n+1}}=0$

$g_{S}(\bar{x}) \prec 0, \quad s \in L \subset\{1,2, \ldots, r\}$

4. NUMERICAL EXAMPLES

4.1 Example

Consider the following biobjective nonlinear problem:

$$
\begin{aligned}
& \min \left(x_{1}, x_{2}\right)^{T} \\
& \text { s.t } \\
& \left(x_{1}-3\right)^{2}+\left(x_{2}-2\right)^{2} \leq 4 \\
& -2 x_{1}+x_{2} \leq 0
\end{aligned}
$$

the Single Objective Problem of original (VOP) using our combined approach can take the following form:

$\min \left(z+x_{1}+u_{2} x_{2}\right)$

s.t

$\beta_{1}\left(x_{1}-1\right)-z \leq 0$, where $f_{1 \text { ideal }}=1$

$\beta_{2}\left(x_{2}-0\right)-z \leq 0$, where $f_{2 i d e a l}=0$

$\left(x_{1}-3\right)^{2}+\left(x_{2}-2\right)^{2} \leq 4$

$-2 x_{1}+x_{2} \leq 0$

Let $u_{2}=.25, \beta_{1}=1 \quad, \beta_{2}=.8$

and use MATLAB program to

solve this SOP problem

Then $\mathrm{x}_{1}=1.522, \mathrm{x}_{2}=0.652, \mathrm{z}=0.522$

\section{To get another solution}

$$
\begin{aligned}
& \text { Let } u_{2}=.5, \beta_{1}=1, \beta_{2}=.8 \\
& \text { use MATLAB program } \\
& \mathrm{x}_{1}=1.522, \quad \mathrm{x}_{2}=0.6525 \quad, \mathrm{z}=0.522
\end{aligned}
$$

To get another solution

Let $u_{2}=2, \beta_{1}=1, \beta_{2}=.8$

$\mathrm{x}_{1}=1.5858, \mathrm{x}_{2}=0.5858 \quad, \mathrm{z}=0.5858$

Let $u_{2}=2, \beta_{1}=5, \beta_{2}=3$

$x_{1}=1.4452, \quad x_{2}=0.742, \quad z=2.226$

Let $u_{2}=10, \beta_{1}=5, \beta_{2}=3$

$\mathrm{x}_{1}=1.971, \quad \mathrm{x}_{2}=0.285$,

$$
\mathrm{z}=4.855
$$

Let $u_{2}=10, \beta_{1}=1, \beta_{2}=.8$

$\mathrm{x}_{1}=2.6078, \quad \mathrm{x}_{2}=0.0388$,

Let $u_{2}=100, \beta_{1}=1 \quad, \beta_{2}=.8$

$\mathrm{z}=1.6078$

$\mathrm{x}_{1}=2.96, \quad \mathrm{x}_{2}=0.0004$,

Let $u_{2}=100, \beta_{1}=.05, \beta_{2}=100$

$\mathrm{x}_{1}=2.979, \quad \mathrm{x}_{2}=0.0001$

$$
z=1.96
$$

$\mathrm{z}=0.0990$

all the above solutions are efficient solutions of the original (VOP)

Characterization of the stability set of the first kind.
Applying Kuhn Tucker condition (K.T.C.) we have Differentiate w.r.to $\mathrm{z}$ :

$$
1-\lambda_{1}-\lambda_{2}=0
$$

Differentiate w.r.to $x_{1}$ :

$1+\lambda_{1} \beta_{1}+2 * v_{1}\left(\bar{x}_{1}-3\right)-2 * v_{2}=0$

Differentiate w.r.to $x_{2}$ :

$$
\begin{aligned}
& u_{2}+\lambda_{2} \beta_{2}+2 * v_{1}\left(\bar{x}_{2}-2\right)+v_{2}=0 \\
& \lambda_{1} \beta_{1}\left(\bar{x}_{1}-1\right)-\lambda_{1} \bar{z}=0, \\
& \lambda_{2} \beta_{2}\left(\bar{x}_{2}-0\right)-\lambda_{2} \bar{z}=0, \\
& v_{1}\left[\left(\bar{x}_{1}-3\right)^{2}+\left(\bar{x}_{2}-2\right)^{2}-4\right]=0 \\
& v_{2}\left[-2 \bar{x}_{1}+\bar{x}_{2}\right]=0 \\
& \left(\bar{x}_{1}-3\right)^{2}+\left(\bar{x}_{2}-2\right)^{2}-4 \prec 0 \\
& , S \in L \subset\{1,2\} \\
& \left(\bar{x}_{1}-3\right)^{2}+\left(\bar{x}_{2}-2\right)^{2}-4=0 \\
& , S \notin L \\
& -2 \bar{x}_{1}+\bar{x}_{2} \prec 0 \\
& , S \in L \subset\{1,2\} \\
& -2 \bar{x}_{1}+\bar{x}_{2}=0 \\
& , S \notin L
\end{aligned}
$$

CASE 1: Let $v_{1}=0$, then $v_{2} \succ 0$, then the above equations become

$\left(\bar{x}_{1}-3\right)^{2}+\left(\bar{x}_{2}-2\right)^{2}-4 \prec 0 \quad, S \in L=\{1\}$

$-2 \bar{x}_{1}+\bar{x}_{2}=0 \quad, S \notin L$

$u_{2}+\lambda_{2} \beta_{2}=-v_{2}$, where $v_{2} \succ 0$, then:

$\left(u_{2}+\lambda_{2} \beta_{2}\right) \prec 0$

$1+\lambda_{1} \beta_{1}=2 * v_{2}$, where $v_{2} \succ 0$,

then: $\left(1+\lambda_{1} \beta_{1}\right)>0$

CASE 2: Let $v_{2}=0$, then $v_{1} \succ 0$, then:

$\left(\overline{\mathrm{x}}_{1}-3\right)^{2}+\left(\overline{\mathrm{x}}_{2}-2\right)^{2}-4=0 \quad, \mathrm{~S} \notin \mathrm{L}$

$-2 \overrightarrow{\mathrm{x}}_{1}+\overline{\mathrm{x}}_{2} \prec 0 \quad, \mathrm{~S} \in \mathrm{L}=\{2\}$

$\mathrm{u}_{2}+\lambda_{2} \beta_{2}=-2 *\left(\bar{x}_{2}-2\right) \quad$ where $\mathrm{v}_{1}>0$

(Depending on the value of $\bar{x}_{2}$ )

$1+\lambda_{1} \beta_{1}=-2 *\left(\overline{\mathrm{x}}_{1}-3\right)$

(Depending on the value of $\bar{x}_{1}$ )

Substitute

$\left(\bar{x}_{1}, \bar{x}_{2}\right)^{t}=(1.5220,0.6525), \quad \bar{z}=.522$

Equations (25-35) we get Linear independent system of equations as follow:

$1-\lambda_{1}-\lambda_{2}=0$

$1+\lambda_{1} \beta_{1}-2.956 v_{1}-2 v_{2}=0$

$u_{2}+\lambda_{2} \beta_{2}-2.695 v_{1}+v_{2}=0$

$v_{1}[0]=0, \quad \Rightarrow v_{1}>0$

$v_{2}[-2.3915]=0, \quad \Rightarrow v_{2}=0$ 
$\left(\bar{x}_{1}-3\right)^{2}+\left(\bar{x}_{2}-2\right)^{2}-4=0 \quad, s \notin L$

$$
\begin{aligned}
& -2 \bar{x}_{1}+\bar{x}_{2} \prec 0 \quad, s \in L=\{2\} \\
& 1+\lambda_{1} \beta_{1}=2.956 v_{1} \quad \Rightarrow\left(1+\lambda_{1} \beta_{1}\right) \geq 2.956 \\
& u_{2}+\lambda_{2} \beta_{2}=2.695 v_{1} \Rightarrow\left(u_{2}+\lambda_{2} \beta_{2}\right) \geq 2.695 \\
& \left.S=\left\{\lambda_{1} \beta_{1}\right) \geq 1.956,\left(u_{2}+\lambda_{2} \beta_{2}\right) \geq 2.695\right\}
\end{aligned}
$$

\subsection{Example}

$$
\begin{aligned}
& f_{1}=\left(x_{1}-1\right)^{2}+\left(x_{2}-1\right)^{2} \\
& f_{2}=\left(x_{1}-2\right)^{2}+\left(x_{2}-3\right)^{2} \\
& f_{3}=\left(x_{1}-4\right)^{2}+\left(x_{2}-2\right)^{2} \\
& \text { s.t }
\end{aligned}
$$

$x_{1}+2 x_{2} \leq 10, x_{2} \leq 4, x_{1} \geq 0, x_{2} \geq 0$

the Single Objective Problem of original (VOP) using our combined approach can take the following form:

$$
\begin{aligned}
& \min \left(z+\left(x_{1}-1\right)^{2}+\left(x_{2}-1\right)^{2}+\right. \\
& u_{2}\left[\left(x_{1}-2\right)^{2}+\left(x_{2}-3\right)^{2}\right]+ \\
& u_{3}\left[\left(x_{1}-4\right)^{2}+\left(x_{2}-2\right)^{2}\right] \\
& \text { s.t } \\
& \beta_{1}\left[\left(x_{1}-1\right)^{2}+\left(x_{2}-1\right)^{2}-0\right]-z \leq 0, \\
& \text { where } f_{1 \text { ideal }}=0 \\
& \beta_{2}\left[\left(x_{1}-2\right)^{2}+\left(x_{2}-3\right)^{2}-0\right]-z \leq 0, \\
& \text { where } f_{2} \text { ideal }=0 \\
& \beta_{3}\left[\left(x_{1}-4\right)^{2}+\left(x_{2}-2\right)^{2}\right]-z \leq 0, \\
& \text { where } f_{3 i d e a l}=0 \\
& x_{1}+2 x_{2} \leq 10, x_{2} \leq 4, \\
& x_{1} \geq 0, x_{2} \geq 0 \\
& \text { Let } u_{2}=2, u_{3}=3, \\
& \beta_{1}=1, \beta_{2}=2, \beta_{3}=3 \\
& \text { and use MATLAB program } \\
& \text { to solve this } S O P \text { problem } \\
& \text { Then } \mathrm{x}_{1}=2.8004, \mathrm{x}_{2}=2.0391, \mathrm{z}=4.3214 \\
& f_{1}=4.3212, f_{2}=1.564, f_{3}=1.4406 \\
& \text { Let } u_{2}=4, u_{3}=5, \\
& \beta_{1}=1, \beta_{2}=2, \beta_{3}=3 \\
& \text { Then } \mathrm{x}_{1}=2.7792, \mathrm{x}_{2}=2.1878, \mathrm{z}=4.5765 \\
& f_{1}=4.5764, f_{2}=1.2668, f_{3}=1.5256 \\
& \text { Let } u_{2}=4, u_{3}=5, \\
& \beta_{1}=6, \beta_{2}=7, \beta_{3}=8 \\
&
\end{aligned}
$$

Then $\mathrm{x}_{1}=2.4926, \mathrm{x}_{2}=1.9024, \mathrm{z}=18.2538$

$f_{1}=3.0422, f_{2}=1.4474, f_{3}=2.2818$

Let $u_{2}=.9, u_{3}=5$,

$\beta_{1}=6, \beta_{2}=7, \beta_{3}=8$

Then $\mathrm{x}_{1}=2.5744, \mathrm{x}_{2}=1.6374, \mathrm{z}=17.3103$

$f_{1}=2.885 \quad, f_{2}=2.1866, f_{3}=2.1638$

Characterization of the stability set of the first kind. Applying Kuhn Tucker condition (K.T.C.) we have Differentiate w.r.to $\mathrm{z}$ :

$1-\bar{\lambda}_{1}-\bar{\lambda}_{2}-\bar{\lambda}_{3}=0$

Differentiate w.r.to $x_{1}$ :

$2\left(\bar{x}_{1}-1\right)+2 \bar{x}_{2}\left(\bar{x}_{1}-2\right)+2 \bar{x}_{3}\left(\bar{x}_{1}-4\right)+2 \bar{\lambda}_{1} \bar{\beta}_{1}\left(\bar{x}_{1}-1\right)+$

$2 \bar{\lambda}_{2} \bar{\beta}_{2}\left(\bar{x}_{1}-2\right)+2 \bar{\lambda}_{3} \bar{\beta}_{3}\left(\bar{x}_{1}-4\right)+\bar{\nu}_{1}=0$

Differentiate w.r.to $x_{2}$ :

$2\left(\bar{x}_{2}-1\right)+2 \bar{u}_{2}\left(\bar{x}_{2}-3\right)+2 \bar{u}_{3}\left(\bar{x}_{2}-2\right)+2 \bar{\lambda}_{1} \bar{\beta}_{1}\left(\bar{x}_{2}-1\right)+$ $2 \bar{\lambda}_{2} \bar{\beta}_{2}\left(\bar{x}_{2}-3\right)+2 \bar{\lambda}_{3} \bar{\beta}_{3}\left(\bar{x}_{2}-2\right)+2 \bar{v}_{1}+\bar{v}_{2}=0$

$\bar{\lambda}_{1} \bar{\beta}_{1}\left[\left(\bar{x}_{1}-1\right)^{2}+\left(\bar{x}_{2}-1\right)^{2}\right]-\bar{z}=0$,

$\bar{\lambda}_{2} \bar{\beta}_{2}\left[\left(\bar{x}_{1}-2\right)^{2}+\left(\bar{x}_{2}-3\right)^{2}\right]-\bar{z}=0$,

$\bar{\lambda}_{3} \bar{\beta}_{3}\left[\left(\bar{x}_{1}-4\right)^{2}+\left(\bar{x}_{2}-2\right)^{2}\right]-\bar{z}=0$,

$\bar{v}_{1}\left(\bar{x}_{1}+2 \bar{x}_{2}-10\right)=0$,

$\bar{v}_{2}\left(\bar{x}_{2}-4\right)=0$

$\bar{x}_{1}+2 \bar{x}_{2}-10 \prec 0, S \in L \subset\{1,2\}$

$\bar{x}_{1}+2 \bar{x}_{2}-10=0, S \notin L$

$\bar{x}_{2}-4 \prec 0, \quad S \in L \subset\{1,2\}$

$\bar{x}_{2}-4=0, \quad S \notin L$

$\bar{x}_{1} \geq 0, \bar{x}_{2} \geq 0$

$2\left(\bar{x}_{1}-1\right)+2 \bar{u}_{2}\left(\bar{x}_{1}-2\right)+2 \bar{u}_{3}\left(\bar{x}_{1}-4\right)+$

$2 \bar{\lambda}_{1} \bar{\beta}_{1}\left(\bar{x}_{1}-1\right)+2 \bar{\lambda}_{2} \bar{\beta}_{2}\left(\bar{x}_{1}-2\right)+$

$2 \bar{\lambda}_{3} \bar{\beta}_{3}\left(\bar{x}_{1}-4\right)=0$

CASE 1: Let $v_{1}=0$, then $v_{2} \succ 0$,

then the above equations become

$\bar{x}_{1}+2 \bar{x}_{2}-10 \prec 0, S \in L=\{1\}$

$\bar{x}_{2}-4=0 \quad, S \notin L$

$2\left(\overline{\mathrm{x}}_{2}-1\right)+2 \overline{\mathrm{u}}_{2}\left(\overline{\mathrm{x}}_{2}-3\right)+2 \overline{\mathrm{u}}_{3}\left(\overline{\mathrm{x}}_{2}-2\right)+$

$2 \bar{\lambda}_{1} \bar{\beta}_{1}\left(\bar{x}_{2}-1\right)+2 \bar{\lambda}_{2} \bar{\beta}_{2}\left(\bar{x}_{2}-3\right)+$

$2 \bar{\lambda}_{3} \bar{\beta}_{3}\left(\bar{x}_{2}-2\right)=-\bar{v}_{2}$

CASE 2: Let $v_{2}=0$, then $v_{1} \succ 0$, then the above equations become

$\bar{x}_{1}+2 \bar{x}_{2}-10=0, \quad S \notin L$

$\bar{x}_{2}-4 \prec 0, \quad S \in L=\{2\}$ 
$2\left(\bar{x}_{1}-1\right)+2 \bar{u}_{2}\left(\bar{x}_{1}-2\right)+2 \bar{u}_{3}\left(\bar{x}_{1}-4\right)+$

$2 \bar{\lambda}_{1} \bar{\beta}_{1}\left(\bar{x}_{1}-1\right)+2 \bar{\lambda}_{2} \bar{\beta}_{2}\left(\bar{x}_{1}-2\right)+$

$2 \bar{\lambda}_{3} \bar{\beta}_{3}\left(\bar{x}_{1}-4\right)=-\bar{v}_{1}$,

(Depending on the value of $\bar{x}_{1}$ )

$2\left(\bar{x}_{2}-1\right)+2 \bar{u}_{2}\left(\bar{x}_{2}-3\right)+2 \bar{u}_{3}\left(\bar{x}_{2}-2\right)+$

$2 \bar{\lambda}_{1} \bar{\beta}_{1}\left(\bar{x}_{2}-1\right)+2 \bar{\lambda}_{2} \bar{\beta}_{2}\left(\bar{x}_{2}-3\right)+$

$2 \bar{\lambda}_{3} \bar{\beta}_{3}\left(\bar{x}_{2}-2\right)=-2 \bar{v}_{1}$

(Depending on the value of $\bar{x}_{2}$ )

Substiute for $\left(\bar{x}_{1}, \bar{x}_{2}\right)^{t}=(2.8,2)^{\mathrm{t}}, \quad \overline{\mathrm{z}}=4.3 \quad$ in the above equations we get Linear independent system of equations as follow:

$\bar{v}_{1}(-3.2)=0$, then $\bar{v}_{1}=0$

$\bar{v}_{2}(-2)=0$, then $\bar{v}_{2}=0$

$1.6 \bar{u}_{2}-2.4 \bar{u}_{3}+3.6 \bar{\lambda}_{1} \bar{\beta}_{1}+1.6 \bar{\lambda}_{2} \bar{\beta}_{2}-2.4 \bar{\lambda}_{3} \bar{\beta}_{3}=-3.6$

$-2 \bar{u}_{2}+2 \bar{\lambda}_{1} \bar{\beta}_{1}-2 \bar{\lambda}_{2} \bar{\beta}_{2}=-2$

$S=\left\{\begin{array}{l}1.6 \bar{u}_{2}-2.4 \bar{u}_{3}+3.6 \bar{\lambda}_{1} \bar{\beta}_{1}+ \\ 1.6 \bar{\lambda}_{2} \bar{\beta}_{2}-2.4 \bar{\lambda}_{3} \bar{\beta}_{3}=-3.6, \\ -2 \bar{u}_{2}+2 \bar{\lambda}_{1} \bar{\beta}_{1}-2 \bar{\lambda}_{2} \bar{\beta}_{2}=-2\end{array}\right\}$

\section{CONCLUSION}

The aim of this research is to introduce a new combined approach, a study of vector optimization problems, its scalarization forms for determining the set of all efficient solutions. In the present work several combined forms are introduced as new single objective problems to characterize the efficient solutions of vector optimization problem. This fact gives an idea to deduce a unified approach which can handle all types of vector optimization problems. In addition, almost the available approaches can be deduced as special cases from it. The new combined approach, combines the characteristics of both the generalized Tchebycheff norm and Lagrangian multiplier method. Also, qualitative analysis is made for redefining and analyzing the basic notions in parametric convex programs for the new combined approach.

Advantages of our approach:

1. The Tchebycheff norm approach possesses complementary distinguishing characteristics for $\mathrm{K}$ th objective Lagrangian approach, so no need to efficiency test because all the generated solutions are efficient to the original problem.

2. The parameters of this approach can be included in both the objective function and the constraints, this allows the decision maker to control the improvement of objective functions, from the relation between the included parameters.

\section{REFERENCES}

[1] Tind J., and Margaret M. Wiecek, " Augmented Lagrangian and Tchebycheff Approaches in Multiple Objective Programming", Department of Operations Research, University of Copenhang, May, 1998.

[2] Miettinen, K., " Nonlinear Multiobjective Optimization ", Kluwer Academic Publishers, (1999).

[3] Hegazy, A.E., " Multiobjective Programming and its Engineering Applications ", Ph.D. Thesis, Faculty of Engineering \& Technology, ElMenoufia University, Shebin El-Kom, Egypt, (1986).

[4] Bowman V. J., "On the relationship of the Tchebycheff norm and the efficient frontier of multiple-criteria objectives", Lecture Notes in Economics and Mathematical Systems, pp.76-85, (1976).

[5] Choo E.-U. and Atkins D.R., "An interactive algorithm for multicriteria programming", Computers and Operations Research 7(1980) 8187.

[6] Khdour, Asmahan. A. K, "Interactive Approaches for Solving Multicriteria Decision Making Problems", PH. D. Thesis, University College for Women, Ain Shams University, Cairo, Egypt, (2005).

[7] Wendell, R.E. and Lee, D.N., "Efficiency in Multiobjective Optimization Problems", Mathematical Programming, Vol.12, pp.406-414, (1977)

[8] Ragab, A. M., : Study on a Special Classes of Multiobjective Nonlinear Programming Problems", Ph. D. Thesis, Ain Shams University, Cairo, Egypt, (1986).

[9] Hussein, M.L., "On Vector Optimization Problems with Application to Game Theory" Ph.D. Thesis, Faculty of Science, Tanta University, Tanta, Egypt, (1988).

[10] Hamada, M.T., "Study for Recent Approaches in solving Vop's " M. Sc. Thesis, Military Technical College, Cairo, Egypt, (1988).

[11] Awad, M.S., "On Multiobjective Mathematical Programming with an Air Defence Application" Ph.D. Thesis, Military Technical College, Cairo, Egypt, (1988).

[12] Steuer, R. E., and Silverman, J., and Whisman, A. W., "A Combined Tchebycheff / Aspiration Criterion Vector Interactive Multiobjective Programming Procedure", Management Science, Vol. 39, P. 1255-1260, (1993).

[13] Kassem, R.M.M., "Multiobjective Nonlinear Programming Methods and Applications" Ph.D. Thesis, Faculty of Economics and Political Sciences, Cairo University, Cairo, Egypt, (1994). 
[14] Gardiner, L.R., and Steuer, R. E., " Unified Interactive Multiple Objective Programming: An Open Architecture for Accommodating New Procedure", J. Oper. Res. Soc, Vol. 45, No. 12, P. 1456-1466, (1994).

[15] Tarrad, M. M. M. M., "Recent Methods in Solving Multicriteria Decision Making Problems", PH. D. Thesis, University College for Women, Ain Shams University, Cairo, Egypt, (1995).

[16] Kanaya, Z.A., "On Interactive Approaches for Solving Fuzzy Multicriteria Decision Making Problems" Ph.D. Thesis, Faculty of Science, Ain Shams University, Cairo, Egypt, (2000).
[17] Abu-Elnaga, U., " Interactive Approaches for Solving Multicriteria Decision Making Problems", PH. D. Thesis, Ain Shams University, Cairo, Egypt, (2006).

[18] Chankong, V. And Haimes, Y, Y., " Multiobjective Decision Making: Theory and Methodology", North-Holland, New York, (1983).

[19] Osman, M.S.A. , "Characterization of the stability set of the first kind for nondifferentiable parametric nonlinear programming". Proceedings of the Fourth Annual Operations Research Conference, Zagazig, Egypt, P. 375-380, (1978). 